\title{
Therapeutic effects of iNOS inhibition against vitiligo in an animal model
}

\author{
Hamid Mansourpour (1), Katayoun Ziari (2), Sahar Kalantar Motamedi (3), Amin Hassan Poor (4)
}

(1) Shahid Beheshti University of Medical Science, Tehran, Iran and AJA University of Medical Science, Tehran, Iran; (2) Department of Pathology, AJA University of Medical Science, Tehran, Iran; (3) Shahid Beheshti University of Medical Science, Tehran, Iran; (4) Tehran University of Medical Science, Tehran, Iran

This article is distributed under the terms of the Creative Commons Attribution Noncommercial License (CC BY-NC 4.0) which permits any noncommercial use, distribution, and reproduction in any medium, provided the original author(s) and source are credited.

\begin{abstract}
Nitric oxide (NO) is involved in several biological processes, but its role in human melanogenesis and vitiligo need further studies. Previous studies revealed that exposure to UVA and UVB were capable of the inducing nitric oxide production in keratinocytes and melanocytes through the activation of constitutive nitric oxide synthase, whereas inducible nitric oxide synthase overexpression has been reported to play an important role in hyperpigmentary disorders. The aim of this study was to evaluate iNOS inhibitor aminoguanidine (AG) as a therapeutic agent in our mouse model of vitiligo. In this study, male C57BL/6J Ler-vit/vit mice were purchased to evaluate the effect of iNOS inhibitor (aminoguanidine) $(50$ and $100 \mathrm{mg} / \mathrm{kg})$ and L-arginine $(100 \mathrm{mg} / \mathrm{kg})$ in a mouse model of vitiligo induced by monobenzone $40 \%$. Moreover, we used phototherapy device to treat the mice with NBUVB as a gold standard.The findings revealed that monobenzone was capable of inducing depigmentation after 6 weeks. However, aminoguanidine in combination with monobenzone was decrease the effect of monobenzone, while L-arginine play a key role in promoting the effect of monobenzone $(\mathrm{P}<0.001)$. Based on the phototherapy, the efficacy of phototherapy significantly increased by adding L-arginine $(\mathrm{P}<0.05)$. Taken together, we suggest that iNOS inhibitor can be a novel treatment for the prevention and treatment of vitiligo by combination of NBUVB therapy, furthermore; NO agents like L-arginine could also increase the effectiveness of phototherapy. Taken together, this pilot study showed significant repigmentation of vitiligous lesions treated with iNOS inhibitor plus NBUVB therapy, where other aspect including expression of an inducible iNOS, NO and TNF levels remained to be evaluated in mice model.
\end{abstract}

Key Words: Vitiligo, Nitric oxide, iNOS, Monobenzone, Treatment.

Eur J Transl Myol 29 (3): 251-260, 2019

Vitiligo, a skin disease, is a common progressive depigmentation of epidermis that results in the destruction of melanocytes in the depigmented parts of the skin, mucous membrane and retinal wall. As a result, irregular white patches appear in different areas of the body. Vitiligo has been estimated to be one of the most frequent pigmentary diseases, accounting for about $0.1-$ $2 \%$ of the worldwide population..$^{1-3}$ The pathophysiology of this disease is not well understood; however, several theories about the pathomechanism of vitiligo have been previously defined, such as genetic, biochemical, immunological, and environmental theories. ${ }^{4,5}$ On the other hand, increasing evidence indicated that there has a correlation between vitiligo and other autoimmune disease of the endocrine glands, ${ }^{6,7}$ as well as a lymphocytic infiltrate was found to play an important role in development of disease when detected in perilesional skin of actively spreading vitiligo. ${ }^{8}$ Several cell lines has been described to play their role in vitiligo development including lymphocytes, keratinocytes, and endothelial cells, involving in melanocyte migration, proliferation, and differentiation. ${ }^{2}$, 9-11 Increasing evidence supports that vitiligo could be a disorder of the entire epidermal melanin unit. ${ }^{12}$ Especially, keratinocytes has been revealed to contribute to the melanocyte homeostasis and its changes affecting melanocyte dysfunction in vitiligo epidermis. ${ }^{1,13,14}$ Vitiligo has been defined as a multifactorial disease with autocytotoxic, neural, and autoimmune susceptibilities as well as environmental factors. ${ }^{15,16}$ Nitric oxide as an intracellular messenger plays an important role in the synthesis of an enzyme called NO synthase, which consists of three 


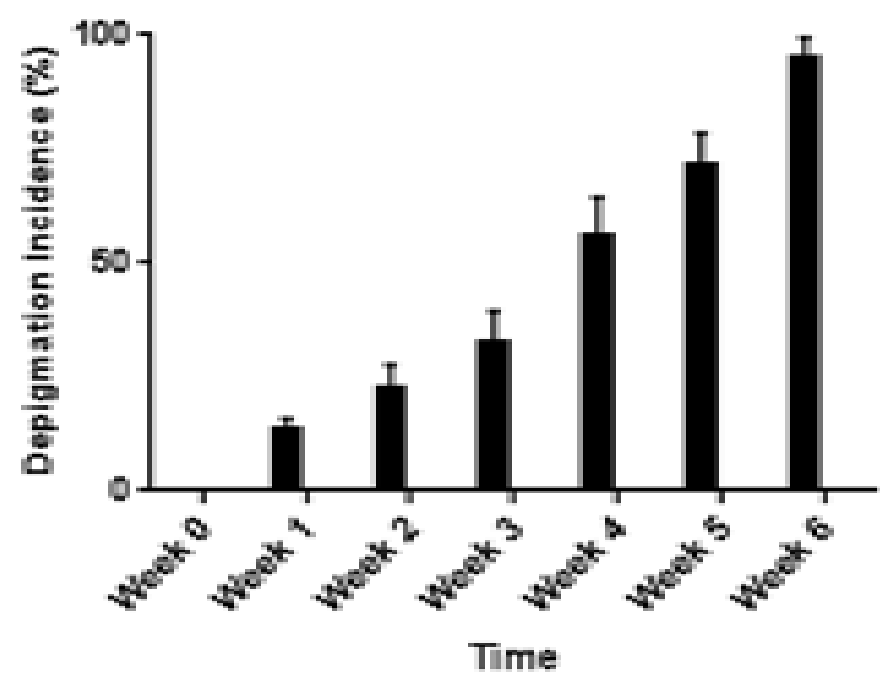

Monobentione-treated group Saline

Fig 1. Monobenzone $40 \%$ induced depigmentation in exposed regions.

isoforms: inducible NOS (iNOS), neuronal NOS (nNOS), and endothelial NOS (eNOS). ${ }^{17-30}$ Nitric oxide has been suggested as a potential factor in inhibiting cell proliferation, differentiation, and apoptosis. This free radical gas can be considered as a great challenge, when a key role plays in pathogenesis of a lot of kind of autoimmune diseases such as vitiligo. Additionally, it is likely to be involved, not only in melanocyte dysfunction and/or destruction, but also in the accumulating toxic intermediates of melanin synthesis and in elevating reactive oxygen species. ${ }^{17-20,22-27} \mathrm{~A}$ growing body of evidence highlights that exposure to UVA and UVB may be capable of producing nitric oxide, especially in keratinocytes and melanocytes, where it is involved, not only in increasing tyrosinase activity via activating constitutive NOS, but also in the synthesis melanin. ${ }^{21}$, ${ }^{31}$ The use of NBUVB can improve treatment of vitiligo by stimulating the proliferation and migration of melanocytes, as well as stimulating the differentiation of melanocyte stem cells. UVA and NBUVB have been introduced as common types of phototherapy in vitiligo. Despite the similar effect of both interventions, narrowband ultraviolet B (NBUVB) phototherapy can be more effective for patients suffering from vitiligo due to the lack of side effects of Psoralen. ${ }^{32,33}$ The topical use of immunosuppressive agents, including glucocorticoids and calcineurin inhibitors, have been introduced as the first line treatment, while the use of phototherapy has been considered as the second line for treating these patients. $^{32}$ iNOS is involved in the production of NO, where promotor polymorphisms of iNOS gene and NO overproduction has been found to be associated with the pathogenesis of vitiligo, leading to melanocyte destruction. $^{34-38}$ Based on above-mentioned data, the current study was aimed to demonstrate the association of vitiligo with iNOS in phototherapy and to investigate the pathophysiology of this disease using a pharmacological inhibition of the iNOS, where a mouse model of vitiligo was induced by monobenzone.

\section{Materials and Method}

\section{Ethics Approvals}

All procedures were performed in accordance with the National Institutes of Health (NIH) Guide for the Care and Use of Laboratory Animals (NIH publication \# 8023) and institutional guidelines for animal care and use (Department of Pharmacology, School of Medicine, TUMS). Each experimental group consisted of 6 to 8 animals.

\section{Animals}

In this study, male C57BL/6J Ler-vit/vit mice were obtained from Pasteur Institute, Tehran, Iran (weighing 25-30 g). Animals were then housed in Plexiglas boxes $(25 \times 25 \times 15 \mathrm{~cm})$, under standard conditions (temperature: $22 \pm 2{ }^{\circ} \mathrm{C}$, humidity: $50 \pm 10 \%$, 12-h lightdark cycle, and free access to food and water).

\section{Phototherapy device}

BIOSKIN®, a phototherapy device is equipped with a short arc generator that provides a beam of visible and ultraviolet radiations, and is also filtered by an interference filter for obtaining UV-B narrow band. The operator is capable of regulating emission time, and plays its role in regulating emission time by accompanying a time-controlled shutter. Furthermore, BIOSKIN ${ }^{\circledR}$ is equipped to prepare a spectrum of intensity up to 400 $\mathrm{mW} / \mathrm{cm}^{2}$, where provide coverage from 300 to $320 \mathrm{~nm}$ for emission spectrum, with an emission peak of $311 \mathrm{~nm}$. Mice were then irradiated three times a week for 4 weeks. The narrow band UV-B (NBUVB) irradiation was performed using a BIOSKIN $®$ device. It is worth noting 


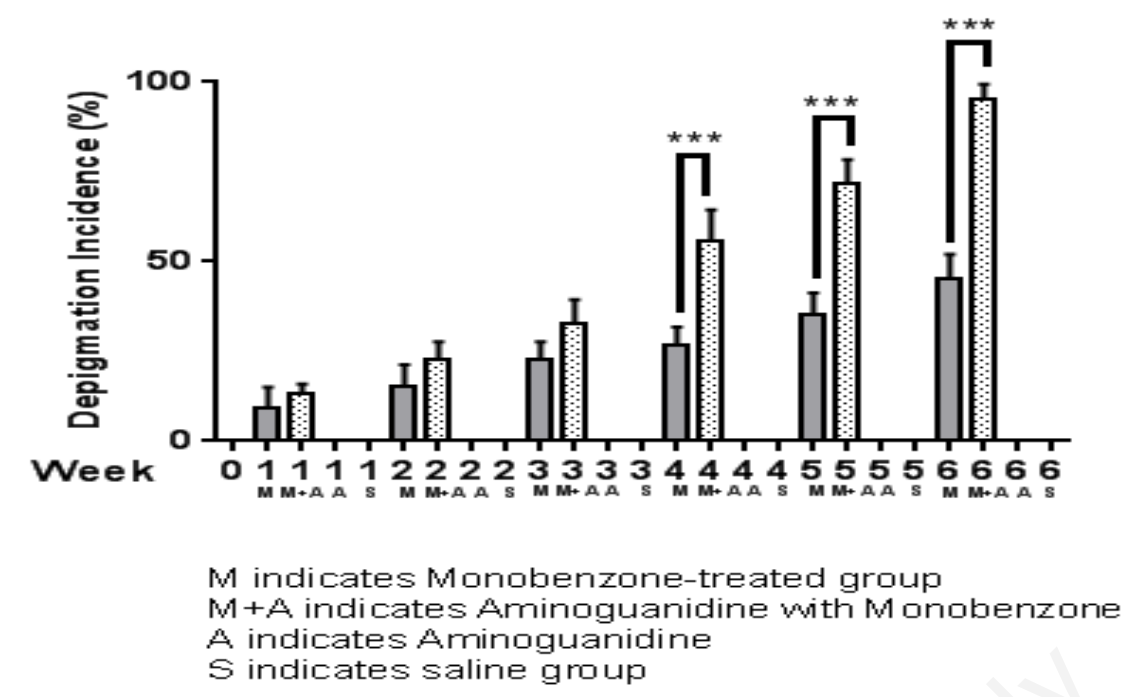

Fig 2. Effect of aminiguanidine (100 $\mathrm{mg} / \mathrm{kg}$ ) plus monobenzone $40 \%$ on depigmentation

that vitiligo patches were next irradiated by excluding mucous membrane and genital part for each microphototherapy session. Although BIOSKIN ${ }^{\circledR}$ equipment is able to provide a large spectrum of intensity (0-400 $\mathrm{mW} / \mathrm{cm}^{2}$ ), the intensity of $50 \mathrm{~mW} / \mathrm{cm}^{2}$ was employed in the present study for all animals during all sessions. The initial dose of irradiation was $20 \%$ less than minimum erythema dose (MED) that was applied to vitiligo area at least 3 days before the beginning of the treatment. During the subsequent sessions, the dose in each session increased to $20 \%$ until the development of erythema. Additional dose of the next session was diminished by $20 \%$ only in the erythematous area when erythema was developed.

\section{Experimental design}

In the present study, sex-matched mice were divided in 6 groups of 10 mice. In the first part of this study we emploied the vitiligo-inducing agent monobenzone in the mice model, as previously described by Zhu et al., ${ }^{39}$ highlightening that monobenzone-induced vitiligo is a valid mouse model of vitiligo. In the first group (control group), mice received only monobenzone cream $40 \%$ on the mouse tail and skin on the back of the mouse and thereafter skin hypo-pigmentations were investigated every week as compared to intact part. In the second group, the mice receive phototherapy (BIOSKIN® device; $50 \mathrm{~mW} / \mathrm{cm}^{2}$ ) in combination with the monobenzone, which was considered a positive control. In the third group of mice, the role of iNOS in development of vitiligo was then evaluated. Therefore, beginning the first day after vitiligo induction animals were treated with aminoguanidine (AG), an injectable iNOS inhibitor, at daily dose of 50 and $100 \mathrm{mg} / \mathrm{kg}$ (i.p. $6 \mathrm{x}$ weekly) to clarify the NO pathway. In group 5 , the combined effect of this inhibitor with phototherapy was investigated. In the sixth group, injectable NO inducer was daily used at dose of $100 \mathrm{mg} / \mathrm{kg}$ (L-arginine) and the combined effect of this drug was investigated by phototherapy. As a matter of fact, the effects of Larginine and $\mathrm{AG}$ on development of vitiligo were assessed by the vitiligo-inducing compound monobenzone. Optimal treatment dose of aminoguanidine was determined by implementing a dose-response model, assessing increasing doses $(50 \mathrm{mg}$ and $100 \mathrm{mg}$ ). All drugs were purchased from Sigma, St Louis, MO, USA and afterward dissolved in saline. Animals received drugs intraperitoneally (i.p.), except for monobenzone.

\section{Results}

\section{Monobenzone induced vitiligo}

In the first step of this study, we tried to evaluate monobenzone 40\%-induced depigmentation from 1 to 6 weeks after exposure. Analysis revealed that monobenzone $40 \%$ can induce vitiligo with a mean of $95 \%$ depigmentation after 6 weeks, which was associated with the increasing trend of vitiligo. One-way ANOVA analysis revealed that monobezone could significantly induce depigmentation after 6 weeks $(F[6,35]=218.2$, $\mathrm{P}<0.001$, Fig1). Post Tukey's analysis showed depigmentation of exposed regions using color cell compression, as given by a computer software after 6 weeks $(\mathrm{P}<0.001)$. As shown in figure 1 , we suggested an increased trend of depigmentation in initiation phase on exposed area.

\section{Co-treatment of monobenzone with iNOS inhibitor}

Following mentioned experiment, we provide a cotreatment of iNOS inhibitor (aminoguanidine at 100 $\mathrm{mg} / \mathrm{kg}$ ) with monobenzone $40 \%$ to clarify the interaction between nitrergic system and vitiligo. Statistical analysis 


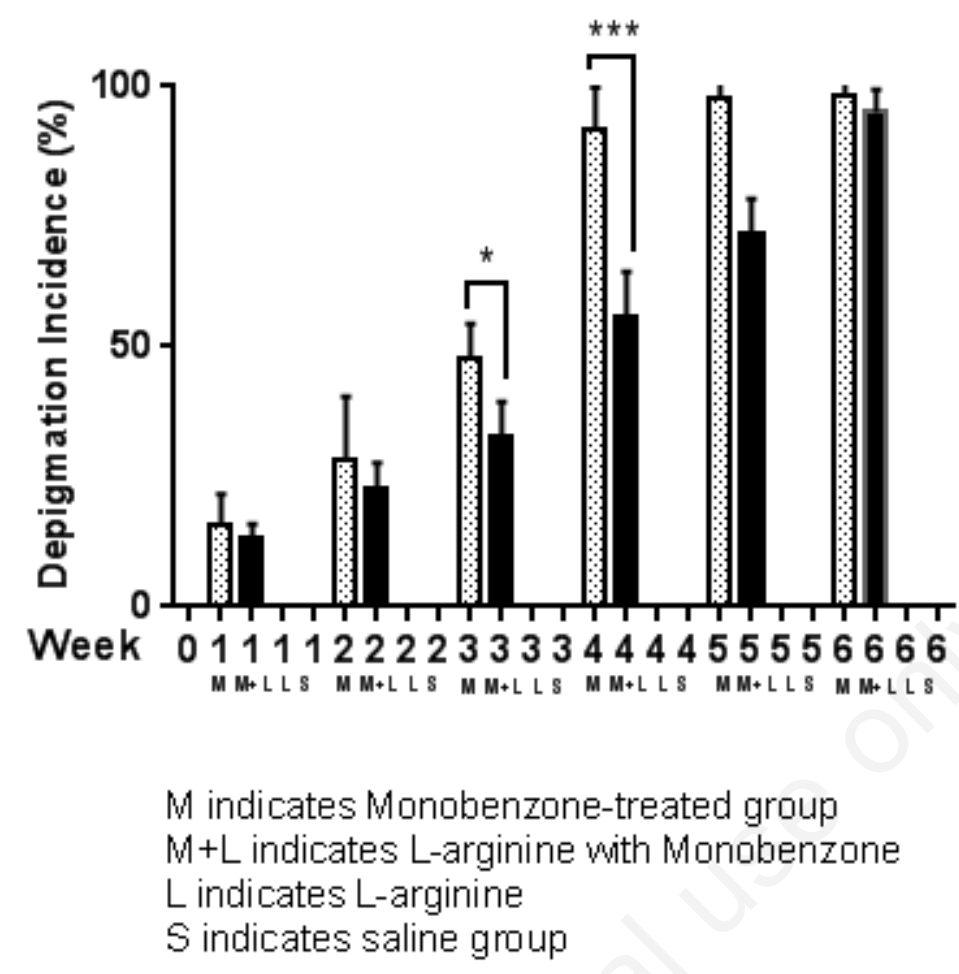

Fig 3. Effect of L-arginine (100 mg/kg) plus monobenzone $40 \%$ on depigmentation.

revealed that co-treatment of aminoguanidine (100 $\mathrm{mg} / \mathrm{kg}$ i.p) with monobenzone $40 \%$ could significantly decrease vitiligo-inducing effect of monobenzone ( $\mathrm{F}$ [12, $65]=127.1 ; \mathrm{P}<0.001 ;$ Fig 2). Post hoc analysis demonstrated a significant difference 4 weeks after treatment $(\mathrm{P}<0.001)$. In addition, the difference between groups was determined as about $50 \%$ of depigmentation after 6 weeks. Overall, aminoguanidine $(100 \mathrm{mg} / \mathrm{kg})$ decreased $50 \%$ of depigmentation after about 6 weeks.

\section{Co-treatment of monobenzone with NO agent}

In the next step, we use NOS agent (L-arginine at 100 $\mathrm{mg} / \mathrm{kg}$ ) in combination with monobenzone $40 \%$ to evaluate the interaction between increasing NO level and vitiligo $(\mathrm{F}[12,65]=181.2 ; \mathrm{P}<0.001 ;$ Fig3 $)$. One-way ANOVA analysis demonstrated that a co-treatment of Larginine $(100 \mathrm{mg} / \mathrm{kg}$ i.p.) with monobenzone $40 \%$ was significantly associated with an increased level of vitiligo-inducing effect of monobenzone. Additionally, post hoc analysis revealed a significant difference between groups after 3 weeks of treatment $(\mathrm{P}<0.05)$. In addition, the difference between groups comprised of $35 \%$ of depigmentation. Furthermore, a significant difference was only observed after 4 weeks of treatment $(\mathrm{P}<0.001)$. Overall, L-arginine $(100 \mathrm{mg} / \mathrm{kg})$ significantly accelerated the depigmentation after about 3 weeks (Figure 3).

\section{Dose dependency of aminiguanidine on inducing vitiligo}

In the next step, we tried to show the dose dependency of aminiguanidine 50 and $100 \mathrm{mg} / \mathrm{kg}$ as previously indicated by our experiments. In order to evaluate the dose dependency of iNOS inhibitor, administration of aminoguanidine (50 and $100 \mathrm{mg} / \mathrm{kg}$ ) with monobenzone $40 \%$ were applied in the current study $(\mathrm{F}[5,30]=273.0$; $\mathrm{P}<0.001$; Fig 4). One-way ANOVA analysis suggested that administration of aminoguanidine $(100 \mathrm{mg} / \mathrm{kg})$ resulted in induction of depigmentation after 6 weeks as compare to aminoguanidine $(50 \mathrm{mg} / \mathrm{kg}),(\mathrm{P}<0.001)$. This experiment showed that aminoguanidine dose dependently could be linked to decreased depigmentation-inducing effect of monobenzone following 6 weeks treatment (Figure 4).

\section{Effect of iNOS inhibitor on phototherapy}

Finally, we evaluated the phototherapy by using NBUVB as the common treatment of vitiligo in combination with L-arginine to clarify the effect of NO level on NBUVB induced pigmentation $(\mathrm{F}[2,15]=10.12 ; \mathrm{P}<0.001$; Fig 5). Analysis revealed that a combination therapy, consisting L-arginine and NBUVB was significantly capable of enhancing the pigmentation of the exposed area, when compared with NBUVB treatment $(\mathrm{P}<0.01)$. Duration of NBUVB radiation therapy is about 4 weeks, where a 


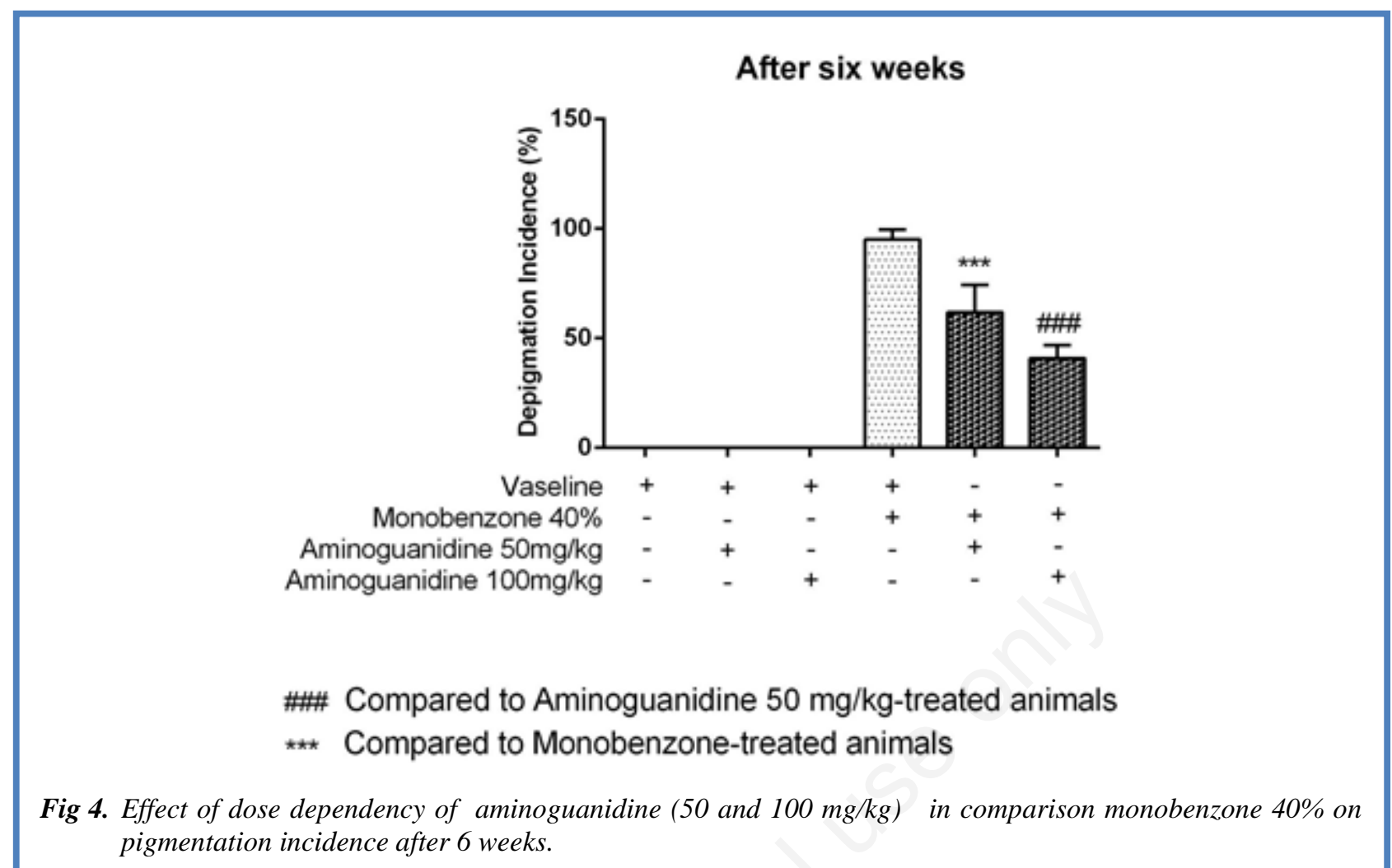

combination therapy of L-arginine and NBUVB increased the effect of NBUVB (Figure 5).

\section{Discussion}

In the current study, we demonstrated that aminoguanidine could inhibit vitiligo-inducing effect of monobenzone after about 4 weeks of treatment. In addition, our results verified that L-arginine could significantly increase the effect of NBUVB treatment in mice model of vitiligo. Nitric oxide has been defined to be a noteworthy mediator that plays a crucial role in numerous biological procedures including melanogenesis, microcirculation, and keratinocyte response to ultraviolet radiation, cell growth and differentiation. Nitric oxide is known to be a major neurotransmitter that plays a key role in the pathophysiology of many diseases of the nervous system, such as epilepsy, schizophrenia, anxiety, and autoimmune disease. Nitric oxide is a chemical messenger that can easily pass through cell membranes, and unlike other neurotransmitters, it does not accumulate in synaptic vesicles and does not release through exocytosis. It seems that nitric oxide plays a crucial role in regulating various body responses, in association with other neurotransmitter systems, such as norepinephrine, serotonin, dopamine, and glutamate. An increasing body of evidence indicates a possible role for NO in hair follicle biology, where is not a well-known regulator in the human hair follicle and the hair-growth cycle. ${ }^{38,39} \mathrm{NO}$ is known as an important mediator of inflammatory responses in the body. Moreover, its precarious role in several hyperproliferative and autoimmune disorders has been previously conformed. ${ }^{40-}$ 43. L-arginine is defined to be a biological precursor of $\mathrm{NO}$, a nerve regulator in the brain, by the Nitric Oxide Synthase (NOS) enzyme. The enzymatic family of NOS consists of three isoforms that include inducible NOS (iNOS), neuronal NOS (nNOS), and endothelial NOS (eNOS). All three NOS isoforms play their crucial role in different parts of the brain. Unlike iNOS, nNOS and eNOS are both calcium / calmodulin-dependent, where preclinical evidence supporting important role for this isoforms in cell homeostasis, and alteration of immune and cytotoxic response $31,44,45$. It is noteworthy, that eNOS and nNOS were originated in numerous tissues, which have been identified to synthesize by different cell including keratinocytes, melanocytes, and fibroblasts in skin. Ample evidence suggests that expression of iNOS is under the regulation of different cytokines including IL-1, IL-6, IL-23, IL-17, IL-33, IFN- $\gamma$ and TNF- $\alpha,{ }^{32,46-}$ 49,50-63 which play a key role in inducing the depigmentation. In this regard, our study should be considered in light of a limitation, where further studies are needed to clarify the expression of cytokines such as TNF-a in the future. IFN- $\gamma$ and TNF- $\alpha$ as Th1 cytokines, because increasing evidence supports a role for Th1 response in mediating vitiligo. A study by Taïeb described vitiligo as an inflammatory disease similar to other skin inflammatory diseases, including psoriasis and atopic dermatitis. This research reported that the elimination of inflammation can be considered as a priority in the treatment of this disease. ${ }^{64}$ 


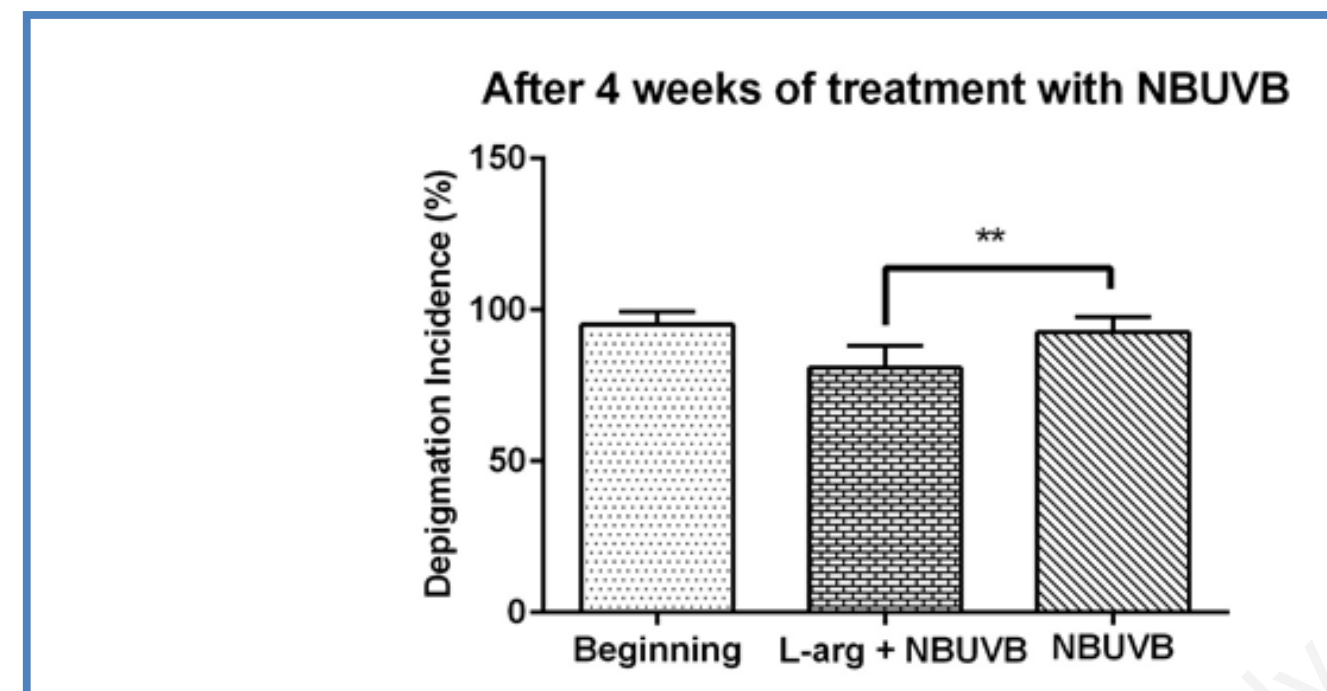

Fig 5. Effect of L-arginine $(100 \mathrm{mg} / \mathrm{kg})$ on pigmentation by using NBUVB therapy.

were capable of triggering the synthesis of NO (NOS) enzymes in keratinocytes and melanocytes. They have suggested that an increased level of NO may increase tyrosine kinase activity and then triggered melanin formation, but excessive increased of inos could lead to processes that result in depigmentation. Aforementioned study exhibited that the NO imbalance play a key role in the development of vitiligo, where inhibitors of this factor can potentially play a role in improving this complication, and recommend the assessment of these inhibitors as a priority. ${ }^{65}$ Many studies suggested the use of topical corticosteroids as the first line of treatment for vitiligo. Moreover, some studies emphasized on the systemic effectiveness of these drugs for reducing inflammation, and symptoms, as well as inducing repigmentation. ${ }^{65}$ On the other hand, immunomodulators (e.g., tacrolimus and calcineurin inhibitors) and reducing the activity of $\mathrm{T}$ cells by decreasing the expression of inflammatory cytokines (e.g., IL-2, IL-3, IL-4, IL-5, interferon (IFN), and TNF) along with the granulocytemacrophage colony-stimulating factor (GM-CSF) can be suggested as effective therapeutic strategies for vitiligo by targeting them that are recently being evaluated. ${ }^{66-67}$ Previous studies revealed that the production of high NO level could be associated with the increased activity of iNOS, confer self-destruction of melanocytes, leading to a decrease in De novo attachment of melanocytes at the extracellular matrix, ${ }^{65,68-70}$ where such evidence can follow skin depigmentation. ${ }^{71}$ Recent mechanism have also attributed to initial imbalance of epidermal cytokines at the affected areas in promoting tetrahydrobiopterin, iNOS activation, and NO overproduction, resulting in an increase of self-destruction of melanocytes. ${ }^{65,72,73}$ In the present study, vitiligo skin was induced by monobenzone $40 \%$, where early findings demonstrated a significant degree of efficacy with appropriate responses, when Larginnine was applied, leading to a decrease in the NO
iNOS inhibitor could decrease the efficacy of monobenzone in the present study. Therefore, emerging evidence has persuaded us to hypothesize that iNOS and NO levels increased in vitiligo lesions compared to healthy controls. In particular, the increased activity of iNOS might be responsible for the overproduction of NO in vitiligo model. Vitiligo is linked to some factors such as increased cytokines expression and iNOS activation that play pivotal roles in the depigmentation process even, if NOS overexpression not being considered as the only important factor involved in vitiligo. An increasing body of evidence indicates that NOS has been overexpressed in vitiligo lesions in comparison with healthy subjects, as well as NO2-/NO3- levels. The increased activity of iNOS has been also suggested to be potentially linked to the increased level of NO in vitiligo, leading to decrease/death of melanocytes up to depigmentation. ${ }^{31}$ Therefore, in the present study, we used an inhibitor to demonstrate whether iNOS inhibition, which is associated with a reduction in NO production, can lead to lesion healing. It is noteworthy that NO increased in vitiligo, as shown preciously by studies. ${ }^{31}$ But its types and possible interventions have not been thoroughly investigated as compared to presented findings herein. On the other hand, evidence from in vivo and in vitro studies supports that the melanocytes can be never entirely disappeared in the depigmented epidermis, where these melanocytes are capable of recovering their function in vivo and in vitro hydrogen peroxide removal. ${ }^{74}$ Moreover, melanocyte survival after some years has been previously demonstrated. ${ }^{75}$ Although measuring NO and TNF levels and viability of skin melanocytes can be useful in confirming the findings, their assessments are considered as part of our limitations. Therefore, further evaluation is needed as a future study, which we hope will be done in the near future. Anyhow, our findings revealed that 
aminoguanidine in combination with monobenzone was able to decrease the effect of monobenzone. The findings indicated that iNOS inhibitor could serve as a novel effective therapy against vitiligo by combination of NBUVB therapy. Furthermore, NO agents like Larginine could markedly increase the effectiveness of phototherapy. Further studies are needed to illustrate expression of an inducible iNOS, NO and TNF levels in mice model for clarification of their role in the pathogenesis of vitiligo. Another approach is necessary to confirm the iNOS implications in the near future: the use of $1400 \mathrm{~W}$. These approaches normalize the "nonspecific effects" of aminoguanidine. An in-vivo siRNA targeting iNOS could be another way to link the iNOS to vitilogous lesions. Systemic infusion of the drugs could interfere with immune profile of the animal. Supporting data for the immune status would strengthen our results and their interpretations.

\section{List of acronyms}

IFN - interferon

GM-CSF - granulocyte-macrophage colony-stimulating factor

NBUVB - narrow band narrowband ultraviolet B

NO - Nitric oxide

NOS - Nitric Oxide Synthase

eNOS - endothelial NOS

iNOS - inducible NOS

nNOS - neuronal NOS

\section{Authors contributions}

KZ, HM, SKM, and AHP conceived and designed the experiments, analyzed the data and wrote the manuscript and supervised the experiments. All authors read and approved the final manuscript.

Acknowledgments None.

\section{Funding None.}

\section{Conflict of Interest}

The authors declare they have no financial, personal, or other conflicts of interest..

\section{Ethical Publication Statement}

We confirm that we have read the Journal's position on issues involved in ethical publication and affirm that this report is consistent with those guidelines.

\section{Corresponding Author}

Katayoun Ziari, Departemnt of Pathology, AJA University of Medical Science, Tehran, Iran. Tel: 00989122993226

Email: drk_ziari@yahoo.com

\section{E-mails of co-authors}

Hamid Mansourpour: mansourpourhamid@yahoo.com Sahar Kalantar Motamedi: skalantarmotamedi@yahoo.com Amin Hassan Poor: amin.hasanzadeh021@gmail.com

\section{References}

1. Ala Y, Pasha MK, Rao RN, et al. Association of IFN-gamma : IL-10 Cytokine Ratio with Nonsegmental Vitiligo Pathogenesis. Autoimmune diseases 2015;2015:423490.

2. Aroni K, Voudouris S, Ioannidis E, et al. Increased angiogenesis and mast cells in the centre compared to the periphery of vitiligo lesions. Arch Dermatol Res 2010;302:601-7.

3. Banerjee P, Venkatachalam S, Mamidi MK, et al. Vitiligo patient-derived keratinocytes exhibit characteristics of normal wound healing via epithelial to mesenchymal transition. Exp Dermatol 2015;24:391-3.

4. Gupta SC, Patchva S, Aggarwal BB. Therapeutic roles of curcumin: lessons learned from clinical trials. AAPS J 2013;15:195-218.

5. Camara-Lemarroy CR, Salas-Alanis JC. The role of tumor necrosis factor-alpha in the pathogenesis of vitiligo. Am J Clin Dermatol 2013;14:343-50.

6. Campanati A, Giuliodori K, Ganzetti G, et al. A patient with psoriasis and vitiligo treated with etanercept. Am J Clin Dermatol 2010;11 Suppl 1:46-8.

7. Barbaud A. What's new in clinical dermatology? Annales de dermatologie et de venereologie 2014;141 Suppl 4:S597-609.

8. Grandemange S, Sanchez E, Louis-Plence P, et al. A new autoinflammatory and autoimmune syndrome associated with NLRP1 mutations: NAIAD (NLRP1-associated autoinflammation with arthritis and dyskeratosis). Ann Rheum Dis 2017;76:1191-8.

9. Vaccaro M, Cicero F, Mannucci C, et al. IL-33 circulating serum levels are increased in patients with non-segmental generalized vitiligo. Arch Dermatol Res 2016;308:527-30.

10. Yao L, Hu DN, Chen M, Li SS. Subtoxic levels hydrogen peroxide-induced expression of interleukin-6 by epidermal melanocytes. Arch Dermatol Res 2012;304:831-8.

11. Yazici AC, Erdal ME, et al. Lack of association with TNF-alpha-308 promoter polymorphism in patients with vitiligo. Arch Dermatol Res 2006;298:46-9.

12. Sandoval-Cruz M, Garcia-Carrasco M, SanchezPorras R, et al. Immunopathogenesis of vitiligo. Autoimmun Rev 2011;10:762-5.

13. Karam RA, Zidan HE, Khater MH. Genetic variants of interferon-gamma and its mRNA expression and inflammatory parameters in the pathogenesis of vitiligo. Biochem Cell Biol 2017;95:474-81.

14. Ning W, Wang S, Dong X, et al. Epigallocatechin3-gallate (EGCG) Suppresses the Trafficking of Lymphocytes to Epidermal Melanocytes via Inhibition of JAK2: Its Implication for Vitiligo Treatment. Biol Pharm Bull 2015;38:1700-6. 
15. Farhan J, Al-Shobaili HA, Zafar U, et al. Interleukin-6: a possible inflammatory link between vitiligo and type 1 diabetes. Brit $\mathbf{J}$ Biomed Sci 2014;71:151-7.

16. Dani P, Patnaik N, Singh A, et al. Association and expression of the antigen-processing gene PSMB8, coding for low-molecular-mass protease 7 , with vitiligo in North India: case-control study. Brit $\mathbf{J}$ Dermatol 2018;178:482-91.

17. Le Poole IC, Stennett LS, Bonish BK, et al. Expansion of vitiligo lesions is associated with reduced epidermal CDw60 expression and increased expression of HLA-DR in perilesional skin. Brit J Dermatol 2003;149:739-48.

18. Marie J, Kovacs D, Pain C, Jouary T, Cota C, Vergier $\mathrm{B}$, et al. Inflammasome activation and vitiligo/nonsegmental vitiligo progression. Brit $\mathrm{J}$ Dermatol 2014;170:816-23.

19. Speeckaert R, Lambert J, Grine L, et al. The many faces of interleukin-17 in inflammatory skin diseases. Brit J Dermatol 2016;175:892-901.

20. Webb KC, Tung R, Winterfield LS, et al. Tumour necrosis factor-alpha inhibition can stabilize disease in progressive vitiligo. Brit $\mathrm{J}$ Dermatol 2015;173:641-50.

21. Zayed AA, Khorshied MM, Hussein MF. Inducible nitric oxide synthase promoter polymorphism: a molecular susceptibility marker for vitiligo in Egyptians. Int J Dermatol 2015;54:675-9.

22. Yeh S, Li Z, Sen HN, et al. Scleritis and multiple systemic autoimmune manifestations in chronic natural killer cell lymphocytosis associated with elevated TCRalpha/beta+CD3+CD4-CD8- doublenegative T cells. Brit J Ophthalmol 2010;94:74852.

23. Manni M, Maestroni GJ. Sympathetic nervous modulation of the skin innate and adaptive immune response to peptidoglycan but not lipopolysaccharide: involvement of betaadrenoceptors and relevance in inflammatory diseases. Brain Behav Immun 2008;22:80-8.

24. Carvalho CL, Ortigosa LC. Segmental vitiligo after infliximab use for rheumatoid arthritis--a case report. Anais brasileiros de dermatologia 2014;89:154-6.

25. Miniati A, Weng Z, Zhang B, et al. Stimulated human melanocytes express and release interleukin8, which is inhibited by luteolin: relevance to early vitiligo. Clin Exp Dermatol 2014;39:54-7.

26. Shin J, Kang HY, Kim KH, et al. Involvement of T cells in early evolving segmental vitiligo. Clin Exp Dermatol 2016;41:671-4.

27. Birlea SA, Costin GE, Norris DA. Cellular and molecular mechanisms involved in the action of vitamin D analogs targeting vitiligo depigmentation. Curr Drug Targets 2008;9:345-59.
28. Bullon P, Navarro JM. Inflammasome as a Key Pathogenic Mechanism in Endometriosis. Curr Drug Targ 2017;18:997-1002.

29. Strassner JP, Harris JE. Understanding mechanisms of autoimmunity through translational research in vitiligo. Curr Opin Immunol 2016;43:81-8.

30. Trcka J, Kunz M. Functional genome and proteome analyses of cutaneous autoimmune diseases. Curr Pharm Des 2006;12:3787-98.

31. Vaccaro M, Irrera N, Cutroneo G, et al. Differential Expression of Nitric Oxide Synthase Isoforms nNOS and iNOS in Patients with Non-Segmental Generalized Vitiligo. Int J Mol Sci 2017;18(12).

32. Ezzedine K, Eleftheriadou V, Whitton M, van Geel N. Vitiligo. Lancet 2015;386(9988):74-84.

33. Esmat S, Mostafa W, Hegazy RA, et al. Phototherapy: The vitiligo management pillar. Clin in Dermatol. 2016;34:594-602.

34. Bhardwaj S, Rani S, Srivastava N, et al. Increased systemic and epidermal levels of IL-17A and IL1beta promotes progression of non-segmental vitiligo. Cytokine 2017;91:153-61.

35. Wang S, Liu D, Ning W, Xu A. Cytosolic dsDNA triggers apoptosis and pro-inflammatory cytokine production in normal human melanocytes. Exp Dermatol 2015;24:298-300.

36. Vaccaro M, Irrera N, Cutroneo G, et al. Differential Expression of Nitric Oxide Synthase Isoforms nNOS and iNOS in Patients with Non-Segmental Generalized Vitiligo. Int J Mol Sci 2017;18:2533.

37. Al-Shobaili HA, Rasheed Z. Mitochondrial DNA acquires immunogenicity on exposure to nitrosative stress in patients with vitiligo. Hum Immunol 2014;75:153-61.

38. Yu N, Zhang S, Sun T, et al. Double-stranded RNA induces melanocyte death via activation of Toll-like receptor 3. Exp Dermatol 2011;20:134-9.

39. Zhu Y, Wang S, Lin F, Li Q, Xu A. The therapeutic effects of EGCG on vitiligo. Fitoterapia 2014;99:243-51.

40. Mitra S, De Sarkar S, Pradhan A, et al. Levels of oxidative damage and proinflammatory cytokines are enhanced in patients with active vitiligo. Free Rad Res 2017;51:986-94.

41. Tsiskarishvili NV, Katsitadze A, Tsiskarishvili NI, Chitanava L. Peculiarities of cytokine status in patients with vitiligo and stress in anamnesis. Georgian medical news 2014;235:45-8.

42. Moretti S, Fabbri P, Baroni G, et al. Keratinocyte dysfunction in vitiligo epidermis: cytokine microenvironment and correlation to keratinocyte apoptosis. Histol Histopathol 2009;24:849-57.

43. Osanto S, Schiphorst PP, Weijl NI, et al. Vaccination of melanoma patients with an allogeneic, genetically modified interleukin 2producing melanoma cell line. Hum Gene Ther 2000;11:739-50. 
44. Lamkanfi M, Vande Walle L, Kanneganti TD. Deregulated inflammasome signaling in disease. Immunol Rev 2011;243:163-73.

45. Vaccaro M, Cannavo SP, Imbesi S, et al. Increased serum levels of interleukin-23 circulating in patients with non-segmental generalized vitiligo. Int J Dermatol 2015;54:672-4.

46. Behfarjam F, Mansouri P, Jadali Z. Imbalance of Peripheral Blood T Helper Type 17 Responses in Patients with Vitiligo. Iranian journal of allergy, asthma, and immunology 2018;17:171-8.

47. Esmaeili B, Rezaee SA, Layegh P, et al. Expression of IL-17 and COX2 gene in peripheral blood leukocytes of vitiligo patients. Iranian journal of allergy, asthma, and immunology. 2011;10:81-9.

48. Strassner JP, Rashighi M, Ahmed Refat M,et al. Suction blistering the lesional skin of vitiligo patients reveals useful biomarkers of disease activity. J Am Acad Dermatol 2017;76:847-55.

49. Pimparkar BD, Bhave A. Arsenicosis: review of recent advances. The Journal of the Association of Physicians of India 2010;58:617-24.

50. Paulos CM, Wrzesinski C, Kaiser A, et al. Microbial translocation augments the function of adoptively transferred self/tumor-specific CD8+ T cells via TLR4 signaling. J Clin Invest 2007;117:2197-204.

51. Barygina V, Becatti M, Lotti T, et al. Treatment with low-dose cytokines reduces oxidativemediated injury in perilesional keratinocytes from vitiligo skin. J Dermatol Sci 2015;79:163-70.

52. Yang Y, Li S, Zhu G, et al. A similar local immune and oxidative stress phenotype in vitiligo and halo nevus. J Dermatol Sci 2017;87:50-9.

53. Moreira CG, Carrenho LZ, Pawloski PL, et al. Preclinical evidences of Pyrostegia venusta in the treatment of vitiligo. J Ethnopharm 2015;168:31525.

54. Roy P, Amdekar S, Kumar A, et al. In vivo antioxidative property, antimicrobial and wound healing activity of flower extracts of Pyrostegia venusta (Ker Gawl) Miers. J Ethnopharma 2012;140:186-92.

55. Garzorz N, Alsisi M, Todorova A, et al. Dissecting susceptibility from exogenous triggers: the model of alopecia areata and associated inflammatory skin diseases. J Eur Acad Dermatol Venereol 2015;29:2429-35.

56. Kim EH, Gasper DJ, Lee SH, Plisch EH, Svaren J, Suresh M. Bach2 regulates homeostasis of Foxp3+ regulatory $\mathrm{T}$ cells and protects against fatal lung disease in mice. J Immunol (Baltimore, Md : 1950). 2014;192:985-95.

57. Li YL, Yu CL, Yu HS. IgG anti-melanocyte antibodies purified from patients with active vitiligo induce HLA-DR and intercellular adhesion molecule-1 expression and an increase in interleukin-8 release by melanocytes. J Inv Dermatol 2000;115:969-73.

58. Morelli JG, Kincannon J, Yohn JJ, et al. Leukotriene $\mathrm{C} 4$ and TGF-alpha are stimulators of human melanocyte migration in vitro. $J$ Inv Dermatol 1992;98:290-5.

59. Redondo P, del Olmo J, Lopez-Diaz de Cerio A, et al. Imiquimod enhances the systemic immunity attained by local cryosurgery destruction of melanoma lesions. J Inv Dermatol 2007;127:167380.

60. Yu HS, Chang KL, Yu CL, et al. Alterations in IL6, IL-8, GM-CSF, TNF-alpha, and IFN-gamma release by peripheral mononuclear cells in patients with active vitiligo. J Inv Dermatol 1997;108:5279.

61. Richmond JM, Bangari DS, Essien KI, et al. Keratinocyte-Derived Chemokines Orchestrate TCell Positioning in the Epidermis during Vitiligo and May Serve as Biomarkers of Disease. The J Inv Dermatol 2017;137:350-8.

62. Lipson EJ, Sharfman WH, Chen S, et al. Safety and immunologic correlates of Melanoma GVAX, a GM-CSF secreting allogeneic melanoma cell vaccine administered in the adjuvant setting. J Transl Med 2015;13:214.

63. Speeckaert R, Lambert J, van Geel N. Clinical Significance of Serum Soluble CD Molecules to Assess Disease Activity in Vitiligo. JAMA Dermatol 2016;152:1194-200.

64. Taïeb A. Vitiligo as an inflammatory skin disorder: a therapeutic perspective. Pigment cell \& melanoma Res 2012;25:9-13.

65. Vaccaro M, Irrera N, Cutroneo G, et al. Differential Expression of Nitric Oxide Synthase Isoforms nNOS and iNOS in Patients with Non-Segmental Generalized Vitiligo. Int J Mol Sci 2017;18:2533.

66. Falabella R, Barona MI. Update on skin repigmentation therapies in vitiligo. Pigment cell \& melanoma Res 2009;22(1):42-65.

67. Grines P. White patches and bruised souls: advances in the pathogenesis and treatment of vitiligo. J Am Acad Dermatol 2004;51:85-7.

68. Rashed L, Abdel Hay R, Mahmoud R, et al. Association of Angiotensin-Converting Enzyme (ACE) Gene Polymorphism with Inflammation and Cellular Cytotoxicity in Vitiligo Patients. PLoS One 2015;10:e0132915.

69. Wang CQ, Cruz-Inigo AE, Fuentes-Duculan J, et al. Th17 cells and activated dendritic cells are increased in vitiligo lesions. PLoS One 2011;6:e18907.

70. Pastor F, Dumas K, Barthelemy MA, et al. Implication of REDD1 in the activation of inflammatory pathways. Sci Rep 2017;7:7023.

71. Levandowski CB, Mailloux CM, et al. NLRP1 haplotypes associated with vitiligo and autoimmunity increase interleukin-1beta 
iNOS inhibition for vitiligo

Eur J Transl Myol 29 (3): 251-260, 2019

processing via the NLRP1 inflammasome. Proc Natl Acad Sci USA 2013;110:2952-6.

72. Wilson SP, Cassel SL. Inflammasome-mediated autoinflammatory disorders. Postgrad Med 2010;122:125-33.

73. Pichler R, Sfetsos K, Badics B, et al. Lymphocyte imbalance in vitiligo patients indicated by elevated CD4+/CD8+ T-cell ratio. Wiener medizinische Wochenschrift (1946). 2009;159:337-41.
74. Tobin DJ, Swanson NN, Pittelkow MR, et al. Melanocytes are not absent in lesional skin of long duration vitiligo. J Pathol 2000;191:407-16.

75. Gottschalk GM, Kidson SH. Molecular analysis of vitiligo lesions reveals sporadic melanocyte survival. Int J Dermatol 2007;46:268-72.

Submission: July 9, 2019

Acceptance: July 24, 2019 